\title{
Status and recent results of the Dark Energy Survey
}

\author{
Eusebio Sanchez ${ }^{* \dagger}$ \\ CIEMAT \\ E-mail: eusebio.sanchez@ciemat.es
}

\begin{abstract}
The current standard model of cosmology has a solid observational basis, but lacks a full understanding of nearly $95 \%$ of the matter-energy content of the universe, that corresponds to two exotic entities: dark matter and dark energy. Currently, a large international scientific effort pursues the goal of unveiling the physical nature of the dark components of the Universe. One of the most imprtant projects in this effort is the Dark Energy Survey (DES), an optical and near infrared survey that is imaging $5000 \mathrm{deg}^{2}$ of the southern celestial hemisphere in five broad bandpass filters, to study the properties of the mysterious dark energy. The survey observations started in 2012, with the science verification run. DES will study the dark energy properties using four independent methods: galaxy clusters counts and distributions, weak gravitational lensing tomography, baryon acoustic oscillations and supernovae Ia distances. Obtaining the four measurements from the same data set will allow a strict control of the systematic uncertainties to obtain a robust and precise determination of the cosmological parameters. Here, some recent scientific results of the project, based on science verification data are presented.
\end{abstract}

The European Physical Society Conference on High Energy Physics

5-12 July

Venice, Italy

${ }^{*}$ Speaker.

${ }^{\dagger}$ On behalf of the DES collaboration. 


\section{Introduction}

The current standard model of cosmology is based on a very large set of independent observations, and has a shocking consequence: only a small fraction (around 5\%) of the content of our Universe is ordinary matter. The other $95 \%$ is composed of strange entities called dark matter and dark energy, that have never been produced in laboratories. The existence of the dark matter is inferred from its gravitational pulling in a large range of scales. The main consequence of dark energy is the accelerated expansion of the Universe, that comes from its negative pressure. Whatever the physical nature of dark energy is, it requires the existence of new physics. Either General Relativity is not a complete theory, and fails to describe gravity at cosmological scales, or some mysterious fluid with negative pressure fills the whole Universe. The current understanding of the nature of dark energy is a non-zero value of the cosmological constant. This description fits all the present cosmological observations [1], some of them to a high degree of precision. However, there is still a large room for improvement in the precision of the dark energy measurements, and it is generally expected that these developments will produce the next advance in our understanding of its physical nature. Therefore, a large number of cosmological experiments has already started or is planned for the future, with the goal of making a reality these improvements. One of the most important projects is the Dark Energy Survey (DES) [2].

\section{The Dark Energy Survey}

The main goal of $\mathrm{DES}^{1}$ is to unveil the physical nature of the dark energy, by means of two interleaved surveys. First, an optical to near-infrared survey that is imaging 5000 square degrees of the southern celestial hemisphere using five wide bandpass filters, griz $Y$, up to magnitude $i_{A B}<24$. Second, a time domain griz survey over 30 square degrees to discover supernovae Ia and measure supernovae their light curves. The project is taking data, and will collect a total of 525 nights during five years, from 2013 to 2018. The main instrument of the DES survey is the 520-Megapixel imager DECam [3], mounted at the prime focus of the Blanco 4m telescope at NOAO's Cerro Tololo Inter-American Observatory (Figure 1). The collaboration consists of more than 300 scientists from USA, UK, Spain, Brazil, Switzerland and Germany.

The DECam CCDs have been specially designed at the Lawrence Berkeley National Laboratory to be extremely sensitive at the red part of the spectrum, in order to allow a good detection of high redshift galaxies. With this new instrument, DES will go beyond the reach of any previous wide area survey by virtue of telescope aperture, median seeing and CCD sensitivity, particularly towards the infrared part of the spectrum.

\section{Scientific Objectives and Forecasts}

DES will study the dark energy by means of the measurement of the parameter $\omega$ of the equation of state and its variation with time to a precision level that will be five times better than the current errors. DES will use four independent methods to perform this measurement, that being done with the same system, allow a very strict control of the systematic errors. The four methods

\footnotetext{
${ }^{1}$ http://www.darkenergysurvey.org
} 


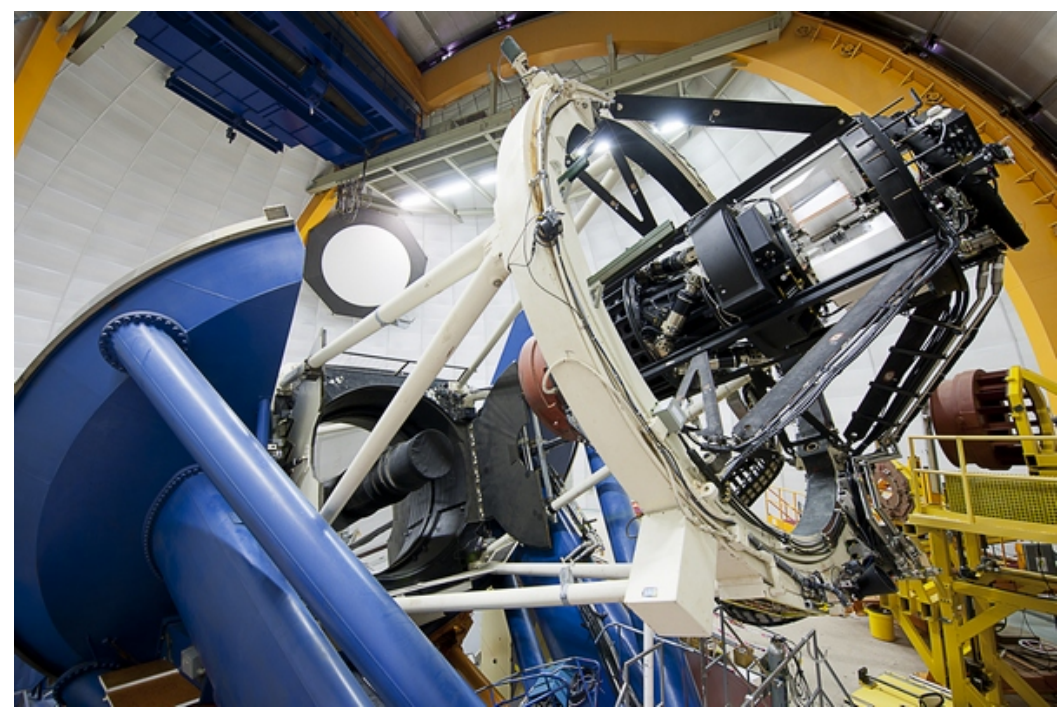

Figure 1: Image of DECam installed at the prime focus of the $4 \mathrm{~m}$ Blanco telescope.

are the following: the counting of galaxy clusters and their spatial distribution at $0.1<z<1$, the measurement of weak lensing shear on several redshift shells up to $z \sim 1$, the determination of the scale of the baryon acoustic oscillations and the spatial distribution of galaxies up to $z<1.4$ and the Hubble diagram for several thousand supernovae Ia at $0.3<z<0.8$.

The expected sensitivity of the DES project for each method is shown in Figure 2, where the equation of state of the dark energy is written as $p / \rho=w_{0}+w_{a}(1-a)$. An improvement of a factor 4 or 5 with respect to the current constraints is expected [6]. However, DES is a very rich scientific project, and will be able to make many other studies. These additional probes will give more constraints and better precision to the final measurement.

\section{Recent Scientific Results}

DES is producing many scientific results, and it is impossible to describe all of them here. Actually, more than 100 papers are already published. We will highlight a few selected results.

\subsection{Dark Matter Distribution in SV Data}

The weak gravitational lensing effect has been measured in the SV data, and the spatial distribution of the projected mass density across the sky has been reconstructed (see Figure 3) using it. This is the largest contiguos mass map ever build, and is a powerful tool for studying cosmology, since it probes both luminous and dark matter [7]. It covers an area of 139 square degrees. The redder regions contain a larger than the average matter density, while the bluer regions are underdense. The overdensities in the reconstructed map correlate well with the distribution of optically detected clusters, depicted as circles, with the size of the circle proportional to the cluster mass. In this work, less than $3 \%$ of the final area that will be mapped by DES ia analysed, so a significant improvement is expected for the next years, while new data sets are considered. Moreover, the SV data have allowed the development of new tools and analysis techniques to build this map. All these techniques will be applied to forthcoming larger data sets from the survey. 


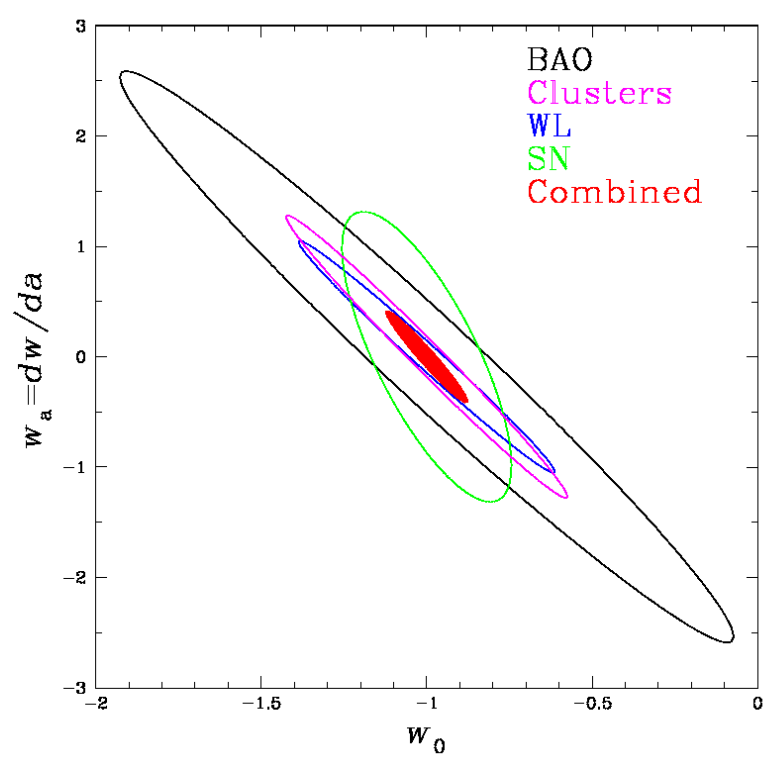

Figure 2: Expected DES sensitivity to dark energy parameters for an equation of state $p / \rho=w_{0}+w_{a}(1-a)$, in each of the measurement techniques: BAO (black), galaxy clusters (magenta), weak lensing (blue) and supernovae Ia (green). The combination of the four methods is also shown (red).

\subsection{Combination of Galaxy Clustering and Weak Lensing for Cosmology}

The DES SV data have allowd to obtain the first cosmological constraints, using a combined analysis of angular clustering of red galaxies and their cross-correlation with weak gravitational lensing of background galaxies. An area of 139 square degrees has been used to perform these measurements, that constrain the matter density of the Universe to be $\Omega_{m}=0.31 \pm 0.09$ and the clustering amplitude of the matter power spectrum to $\sigma_{8}=0.74 \pm 0.13$ after marginalizing over seven nuisance parameters and three additional cosmological parameters.

The robustness of results has been extensively checked, with respect to several variations in the choice of data vector and modelling and treatment of systematics. In particular, the results are stable under the use of two different shear catalogs, four different photo-z codes and two different estimators of the lensing signal. They also show consistency with the fiducial results when using a different lens bin, a different selection of angular scales or when adding a nonlinear galaxy bias parameter.

As has been stated before, the DES-SV region comprises only $3 \%$ of the final survey coverage, and we expect a substantial improvement on the constraining power with future data releases. However, these results are interesting, since they validate the robust modelling of systematic errors and galaxy bias, as well as the exhaustive testing of the shear pipeline, photo-z estimation and the selection of the galaxy sample. All these tests allow the application of the developed techniques to future and more powerful data sets.

\section{Conclusions}

DES is a large galaxy survey that will measure the dark energy properties using four inde- 


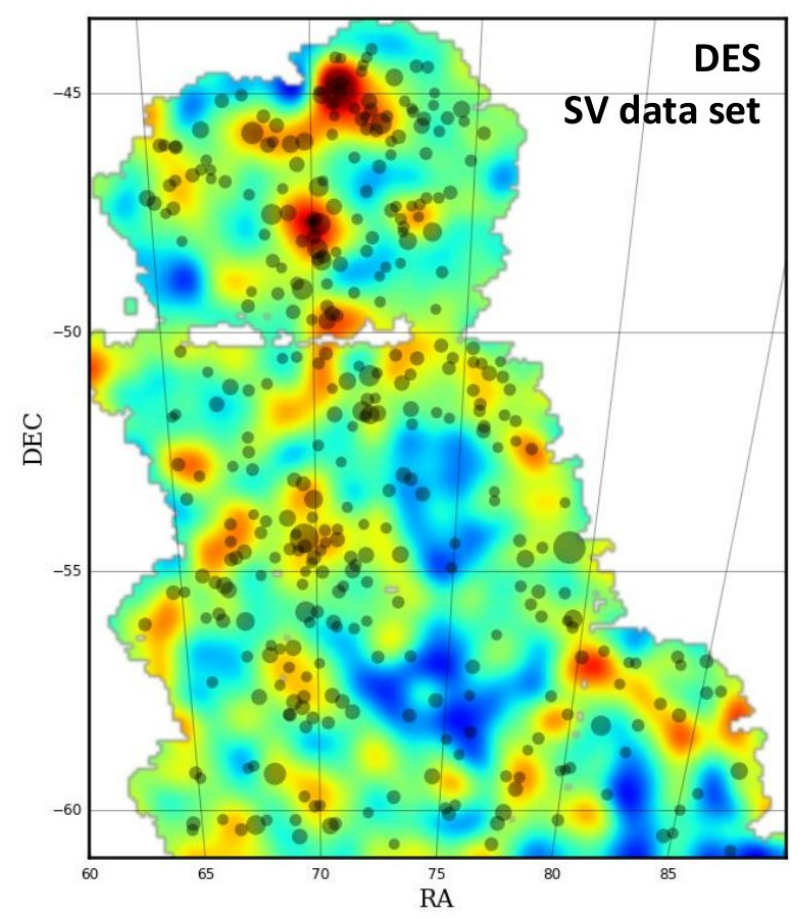

Figure 3: DES SV mass map obtained from the weak lensing convergence. The map is generated with $5 \times 5$ square arcmin pixels and 20 arcmin RMS Gaussian smoothing. Red areas correspond to overdensities and blue areas to underdensities. White regions correspond to the survey mask. The map is overlaid by galaxy clusters, represented as circles. The radius of the circles scale with the cluster mass.

pendent probes: galaxy clusters, baryon acoustic oscillations, weak gravitational lensing and supernovae Ia. Very robust and low systematics measurements are expected, since the multiprobe approach has been designed to improve the current precision by a factor of 4 or 5 .

SV data are of high quality, and have already allowed to obtain a large variety of scientific results, but the data quality and quantity for DES as a whole will be a major step beyond current surveys. Here, some of the most interesting recent results have been presented. These results, in addition, have allowed the development of the tools and methods that will be used in the analysis of the full DES data set. In particular, a mass map of the area that has been covered in the SV data has been obtained. This is the largest continuous mass map up to date. In addition, the first cosmological results that combine galaxy clustering and weak lensing have been obtained for the SV data. This analysis has allowed a determination of the matter density of the Universe and the clustering amplitude of the matter power spectrum to be $\Omega_{m}=0.31 \pm 0.09$ and $\sigma_{8}=0.74 \pm 0.13$ respectively.

\section{Acknowledgements}

Funding support for this work was provided by the Spanish Ministry of Economy and Competitiveness (MINECO) through grant FPA2012-2015-68048-C3-02-P.

Funding for the DES Projects has been provided by the DOE and NSF (USA), MINECO (Spain), STFC (UK), HEFCE (UK), NCSA (UIUC), KICP (U. Chicago), CCAPP (Ohio State), 
MIFPA (Texas A\&M), CNPQ, FAPERJ, FINEP (Brazil), DFG (Germany) and the Collaborating Institutions in the Dark Energy Survey.

The Collaborating Institutions are Argonne Lab, UC Santa Cruz, University of Cambridge, CIEMAT-Madrid, University of Chicago, University College London, DES-Brazil Consortium, University of Edinburgh, ETH Zürich, Fermilab, University of Illinois, ICE (IEEC-CSIC), IFAE Barcelona, Lawrence Berkeley Lab, LMU München and the associated Excellence Cluster Universe, University of Michigan, NOAO, University of Nottingham, Ohio State University, University of Pennsylvania, University of Portsmouth, SLAC National Lab, Stanford University, University of Sussex, Texas A\&M University, and the OzDES Membership Consortium.

Based in part on observations at Cerro Tololo Inter-American Observatory, National Optical Astronomy Observatory, which is operated by the Association of Universities for Research in Astronomy (AURA) under a cooperative agreement with the National Science Foundation.

The DES Data Management System is supported by the NSF under Grant Numbers AST1138766 and AST-1536171. The DES participants from Spanish institutions are partially supported by MINECO under grants AYA2015-71825, ESP2015-88861, FPA2015-68048, and Centro de Excelencia SEV-2012-0234, SEV-2016-0597 and MDM-2015-0509. Research leading to these results has received funding from the ERC under the EU's $7^{\text {th }}$ Framework Programme including grants ERC 240672, 291329 and 306478. We acknowledge support from the Australian Research Council Centre of Excellence for All-sky Astrophysics (CAASTRO), through project number CE110001020.

This manuscript has been authored by Fermi Research Alliance, LLC under Contract No. DE-AC02-07CH11359 with the U.S. Department of Energy, Office of Science, Office of High Energy Physics. The United States Government retains and the publisher, by accepting the article for publication, acknowledges that the United States Government retains a non-exclusive, paid-up, irrevocable, world-wide license to publish or reproduce the published form of this manuscript, or allow others to do so, for United States Government purposes.

\section{References}

[1] N. Nakamura et al., J. Phys. G 37 (2010) 075021.

[2] B. L. Flaugher, Int.J.Mod.Phys. A20 (2005) 3121-3123.

[3] B. Flaugher et al., Astron. Jour. 150 (2015) 150.

[4] H. T. Diehl et al., Proc. SPIE, Int. Soc. Opt. Eng. 9149 (2014).

[5] A. Albrecht et al., Report of the Dark Energy Task Force, arXiv:astro-ph/0609591 [astro-ph].

[6] J. Weller, et al., The dark energy survey, Proceedings of Cosmology, Galaxy Formation and Astroparticle Physics on the Pathway to the SKA , H. R. Klöckner editor (2008) ISBN 9789080543447.

[7] C. Chang et al., Phys. Rev. Lett. 115 (2015) 051301.

[8] J. Kwan et al., Mon. Not. R. Astron. Soc 464 (2017) 4045 - 4062. 